\title{
Frontières
}

\section{L'éducation à la perte et au deuil}

\section{Pierre-Alexandre Poirier}

Volume 13, numéro 1, automne 2000

\section{La mort au tableau noir}

URI : https://id.erudit.org/iderudit/1074224ar

DOI : https://doi.org/10.7202/1074224ar

Aller au sommaire du numéro

Éditeur(s)

Université du Québec à Montréal

ISSN

1180-3479 (imprimé)

1916-0976 (numérique)

Découvrir la revue

Citer cet article

Poirier, P.-A. (2000). L'éducation à la perte et au deuil. Frontières, 13(1), 40-44. https://doi.org/10.7202/1074224ar

\section{Résumé de l'article}

Nous pensons que les enseignants du primaire peuvent être formés pour prendre en main une situation de deuil dans la classe. Cela ne signifie certes pas que l'enseignant doive assumer les fonctions du psychologue, de l'infirmière, du prêtre ou du travailleur social. Sa pratique professionnelle le tient très occupé et il ne saurait s'improviser spécialiste du deuil. En revanche, quand survient un accident grave ou le décès d'un élève ou d'un membre de l'équipe-école, l'enseignant est assurément le mieux placé pour accueillir et traiter les premières réactions des élèves. Dans cet article, nous présentons des connaissances utiles à un enseignant désirant mieux comprendre le deuil dans l'enfance, et quelques pistes d'intervention.
Ce document est protégé par la loi sur le droit d'auteur. L'utilisation des services d'Érudit (y compris la reproduction) est assujettie à sa politique d'utilisation que vous pouvez consulter en ligne.

https://apropos.erudit.org/fr/usagers/politique-dutilisation/ 


\section{Résumé}

Nous pensons que les enseignants du primaire peuvent être formés pour prendre en main une situation de deuil dans la classe. Cela ne signifie certes pas que l'enseignant doive assumer les fonctions du psychologue, de l'infirmière, du prêtre ou du travailleur social. Sa pratique professionnelle le tient très occupé et il ne saurait s'improviser spécialiste du deuil. En revanche, quand survient un accident grave ou le décès d'un élève ou d'un membre de l'équipe-école, l'enseignant est assurément le mieux placé pour accueillir et traiter les premières réactions des élèves. Dans cet article, nous présentons des connaissances utiles à un enseignant désirant mieux comprendre le deuil dans l'enfance, et quelques pistes d'intervention.

Mots clés : deuil - enfant - rituel

\begin{abstract}
We think that primary school teachers can be trained to help children mourn the loss of fellow classmates. This does not imply that teachers should assume the function of psychologists, nurses, priests or social workers. Teachers are already weighted by classroom duties and it cannot be expected that they become specialists of mourning. However, when a grave accident or death occurs among one of the pupils, the teacher is more likely to observe the first reactions to loss among them. In this article, we present useful information to the teacher desiring to understand mourning among children, and some reflections on ways of assisting.
\end{abstract}

Key words : mourning - child - ritual

\section{L'éducation à la perte et au deuil}

\author{
Pierre-Alexandre Poirier, \\ doctorant en sociologie de l'éducation, \\ Sorbonne - Université Laval.
}

Si l'étude du deuil chez les enfants est relativement récente, plus récentes encore sont les recherches portant sur le deuil et la mort en contexte scolaire. Au Québec, l'Église a longtemps été omniprésente dans l'école. Maintenant, le psychologue et le travailleur social remplacent souvent le prêtre auprès des enfants endeuillés. En plus des interventions ponctuelles du psychologue et du travailleur social, il y a lieu de se demander si les enseignants pourraient être mieux préparés pour aider un élève en deuil.

\section{LE DEUIL ET SES SOUFFRANCES}

Faire une éducation au deuil consiste à former les enseignants afin qu'ils puissent prendre en main une situation de deuil dans la classe. Cela ne signifie certes pas que l'enseignant doive assumer les fonctions du psychologue, de l'infirmière, du prêtre ou du travailleur social. Sa pratique professionnelle le tient très occupé et il ne saurait s'improviser spécialiste du deuil. En revanche, quand survient un accident grave ou le décès d'un élève ou d'un membre de l'équipe-école, l'enseignant est assurément le mieux placé pour accueillir et traiter les premières réactions des élèves.

Les différents contextes dans lesquels une personne peut être en deuil ont tous un point commun : la souffrance. Le mot "deuil » vient du latin dolere qui veut dire "souffrir». Une personne en deuil est une personne qui souffre. Or, la souffrance du deuil est toujours un peu mystérieuse. On ne sait pas comment la souffrance du deuil atteint une personne, on ne sait pas comment elle la vit, comment elle va l'exprimer'. Il se peut que des personnes cherchent à éviter de vivre la souffrance du deuil parce qu'elles ont peur de leur fragilité, de leur solitude, de leur faiblesse. Elles ont peur également de montrer une image d'elle-même qu'elles n'aiment pas, qui les met mal à l'aise. D'autres personnes vont se détourner du deuil parce qu'elles ont peur du ridicule, elles ont peur que la manifestation de la souffrance les dévalorise. À vrai dire, il n'y a rien de déshonorant à vivre et à exprimer sa souffrance.

La souffrance du deuil, en fait, est plurielle du fait qu'elle pourra être 
éprouvée notamment comme rage, comme ennui, comme impuissance, comme peur, comme colère, comme consternation, comme affliction, comme déception, comme trahison, comme tristesse. C'est pourquoi la souffrance du deuil est une expérience extrêmement complexe. À bien des égards, le deuil des enfants n'est pas moins complexe que celui des adultes.

Selon Michel Hanus², le sens du mot " deuil » s'est aujourd'hui élargi à trois réalités distinctes. Le deuil désigne en premier lieu la souffrance d'une personne confrontée à une perte. Parler du deuil en ce sens renvoie à l'état affectif de l'endeuillé, c'est-à-dire à la peine ou à la douleur de vivre la perte d'un objet aimé. Ensuite, le deuil désigne l'ensemble des rituels accomplis pour négocier les sentiments suscités par une perte. Une personne confrontée à une perte devra ritualiser des sentiments de désarroi, de rage, de colère, d'injustice, d'impuissance, de souffrance. Finalement, le deuil désigne le travail psychique ${ }^{3}$ pour se détacher de l'objet aimé (personne, chose, emblème, idéaux, etc.). Il s'agit ici d'apprendre à ne plus souffrir de l'objet perdu tout en lui conservant une place de choix dans ses souvenirs.

Le deuil sera vécu par un seul élève ou encore par l'ensemble de la classe. On peut faire une distinction entre le deuil occasionné par une perte individuelle et le deuil occasionné par une perte collective ${ }^{4}$. À n'en point douter, les enfants sont appelés, au cours de leur re ou d'un camarade de classe. Dans ce type de situation, c'est l'ensemble des élèves de la classe qui sera profondément affecté.

Qu'il s'agisse d'une perte intime ou d'une perte collective, le deuil est toujours personnel, c'est-à-dire que chacun est seul dans sa souffrance. L'entourage peut certes être présent pour soutenir le moral et offrir une aide fraternelle. Toutefois, personne ne peut vivre la souffrance d'un deuil à la place d'un autre. Le caractère unique de chaque individu et la spécificité des liens qui l'unissent au défunt singularisent sa souffrance.

Lorsqu'une classe est confrontée à une perte particulièrement bouleversante, l'enseignant doit lui-même prendre une distance face à sa propre souffrance pour aider les élèves. On ne saurait pousser des enseignants à assumer des situations de souffrance qui débordent leur capacité. Le fait de parler de l'événement témoigne déjà d'une certaine capacité de le prendre en charge. Même l'enseignant qui avoue son incapacité à en parler montre qu'il peut, à sa façon et dans une certaine mesure, acquérir une certaine maîtrise sur la situation. Parce qu'il a le courage d'avouer son impuissance, il indique aux élèves que la souffrance du deuil n'est pas nécessairement facile à vivre et à exprimer. C'est justement ce que l'enfant veut voir chez un adulte. La manière dont l'adulte agit, en fait, est l'une des assises à partir de laquelle l'enfant va élaborer son propre vécu.

\section{QU'IL S'AGISSE D'UNE PERTE INTIME OU D'UNE PERTE COLLECTIVE,}

LE DEUIL EST TOUJOURS PERSONNEL, C'EST-À-DIRE QUE CHACUN EST SEUL DANS SA SOUFFRANCE.

vie, à connaître un grand nombre de deuils personnels : l'enfance qui s'envole, un ami qui déménage, un changement de classe ou d'enseignant, une personne particulièrement appréciée qui trahit ou qui déçoit, un amour secret qui est refusé, un projet qui avorte, un mauvais résultat scolaire, etc. Tous ces événements sont pour un élève des occasions de faire des deuils intimes qui passent inaperçus aux yeux des autres élèves, mais qui laissent parfois des traces indélébiles. D'autres deuils seront vécus en groupe : ils peuvent être causés par le décès d'un membre du personnel scolai- laires. La vie de la classe lui semblera pour un moment beaucoup plus futile. L'élève confronté à une perte éprouve également des difficultés à répondre correctement aux exigences relatives aux travaux scolaires demandés par son enseignant. Si ce dernier comprend que l'enfant est pour l'instant incapable de s'investir dans son travail scolaire, il peut sans aucun doute mieux comprendre pourquoi il a besoin d'une aide. Perdre une personne aimée est une expérience très difficile, il est inutile d'ajouter à celleci les réprobations d'un enseignant mécontent du travail de l'élève.

\section{LE DEUIL ET L'ENFANT}

On ne peut nier la difficulté pour les parents et les spécialistes du deuil d'évaluer les effets d'une perte importante pour l'enfant. De façon générale, deux éléments doivent être pris en compte. L'adulte peut d'abord surestimer l'importance d'une perte chez les enfants et croire qu'ils seront d'emblée malheureux, déprimés, perturbés ou asociaux. Il peut, a contrario, sous-estimer l'importance d'un deuil précoce et rester aveugle au besoin d'aide et de réconfort que ressent l'enfant. Entre ces deux extrêmes, il est parfois difficile pour les parents et les enseignants de saisir les sentiments de l'enfant, c'est-à-dire de tenter de comprendre la charge émotive liée à la perte.

Françoise Dolto affirme qu'il n'y a pas d'âge pour être bouleversé par une perte importante. Dans Tout est langage, elle écrit : "Il n'y a pas d'âge pour le dire [le décès]. On peut dire cela à huit jours, à quinze jours, il n'y a pas d'âge, il faut que ce malheur soit dit, tel que les parents responsables de l'enfant l'éprouvent. C'est comme cela que nous l'aidons le mieux. ${ }^{6}$ ". Michel Hanus note que l'enfant est en mesure de travailler une perte importante à partir de trois ou quatre $\mathrm{ans}^{7}$. Un enfant est jugé apte à faire un travail de deuil lorsqu'il est en mesure de se détacher d'un objet perdu ayant été préalablement investi d'une importante quantité de libido. En fait, il affirme que le déroulement du deuil de l'enfant est partiellement semblable à celui de l'adulte en ce qu'il se schématise selon la même séquence de refus, de dépression et d'adaptation. Toutefois, ce travail de deuil reste sous plusieurs aspects très différent de celui de l'adulte. En effet, l'enfant doit encore faire certaines acquisitions d'ordre intellectuel, affectif et cognitif avant de pouvoir faire un réel travail de deuil : maîtrise suffisante de la réalité de la mort, acquisition d'une représentation interne stable de 
l'objet perdu et certitude que ses besoins physiologiques et psychologiques seront satisfaits en l'absence du parent perdu.

\section{RÉALITÉ ET FICTION}

Parce que l'enfant est en pleine période de croissance, les deuils qu'il peut vivre sont quelquefois compliqués. À la différence de l'adulte, l'enfant est moins apte à faire la distinction entre ses propres désirs et la réalité. Habituellement, un adulte confronté à une perte difficile sait faire une différence entre ses désirs, qui le poussent à imaginer que la personne aimée est toujours présente, et le drame de la réalité qui l'oblige à reconnaître que cette même personne est décédée. Chez l'enfant, cette capacité est encore fragile. Il y a chez lui une fragile cohabitation entre la partie de son moi qui reconnaît la réalité et l'autre qui la refuse.

Il appartient à l'enseignant comme à n'importe quel adulte en qui l'enfant met sa confiance de l'aider à donner un statut à la personne disparue. L'enseignant peut ainsi contribuer à éviter que l'enfant idéalise le défunt de manière excessive. Il appartient également à l'enseignant d'aider l'enfant à se libérer de ses sentiments de culpabilité. Les enfants vivent dans un sentiment d'omnipotence et de toute-puissance qui les porte à croire qu'ils peuvent être responsables de tous les malheurs de leur entourage. Les adultes doivent les convaincre qu'ils n'ont pas causé la mort d'un être aimé.

\section{VÉRITÉ ET CULPABILITÉ}

Certains enseignants peuvent chercher à ménager les enfants en leur cachant la vérité du décès. La plupart du temps, les enfants connaissent la vérité, ou ils en ont entendu parler sans en être certain. Ils ressentent durement le silence autour de la réalité d'une perte et l'interprètent comme un manque de confiance des adultes à leur égard. Pour eux, ce manque de confiance peut confirmer leur part de responsabilité dans la tragédie. Comment comprendre les sentiments de culpabilité d'un enfant en deuil ? La culpabilité s'explique par le narcissisme de l'enfant et par une ambivalence des sentiments lors de l'investissement d'objet. Le narcissisme est une illusion qui confère un sentiment de toute-puissance à l'enfant. Cette toutepuissance lui laisse croire qu'il a un certain " pouvoir magique " pour agir sur les objets de son entourage. Il peut penser qu'il est à l'origine d'un événement qui vient de se produire. De plus, il se sent responsable du fait qu'il aurait déjà peut-être souhaité la mort du défunt. Ce désir inconscient de mort est discuté par Freud dans $A u$ delà du principe de plai$\operatorname{sir}^{8}$. Le père de la psychanalyse a noté que les investissements libidinaux sont imprégnés de désirs amoureux, tendres et sensuels, mais aussi de désirs agressifs. Freud soutient que tous les objets dans lesquels une personne s'est investie sont à la fois aimés et détestés. Si on ajoute à ces sentiments ambivalents la pensée magique issue du narcissisme, il est possible de penser que celui ou celle qui vient de perdre un parent ou un ami se reproche de ne pas avoir fait tout ce qui était possible pour empêcher qu'un tel drame se produise, ou de n'avoir rien fait pour l'éviter.

\section{LA MORT POUR L'ENFANT}

Pour l'enfant, la mort est un phénomène difficile à comprendre. L'enfant placé devant le décès d'une personne aimée est confronté à la mort avant même d'avoir développé les ressources psychiques lui permettant d'en constituer une représentation ${ }^{9}$. Pour l'enfant comme pour l'adulte, la mort a quelque chose de mystérieux. Or, l'imaginaire relatif à la mort est plutôt pauvre chez l'enfant. En effet, la représentation que se fait un enfant de la mort est davantage liée à des expériences vécues et à des événements de son quotidien tels que la mort de son poisson rouge ou la mort d'une fourmi. Trois traits dominent ses être déconcertés par la réponse d'un enfant qui affirme comprendre que leur maman est décédée et qui, tout de même, désire savoir quand elle reviendra. Troisièmement, la mort est contagieuse. Pour l'enfant, la mort fonctionne comme un mauvais virus pouvant contaminer tous et chacun.

La maîtrise suffisante de la réalité de la mort est indispensable à la mise en place d'un travail de deuil. Or, une telle maîtrise est le fruit d'une longue évolution des représentations de la mort que se font les enfants. Ces représentations sont au départ bien différentes de celles des adultes et évoluent avec le temps pour s'y conformer. Chez les enfants, la conception de la mort est davantage liée à la pensée magique tandis que, pour l'adulte, elle est en grande partie liée aux apprentissages réalisés lors de la confrontation avec la réalité.

\section{QUESTIONS D'ENFANT}

Deux questions sur la mort reviennent souvent dans les paroles des enfants. Ce sont deux questions plutôt embêtantes qui méritent qu'on s'y attarde. Les enfants se demandent s'il est vrai qu'on meurt comme ça, tout de suite. Dolto $^{11}$ suggère de répondre que c'est vrai, que ça peut arriver. C'est la vérité, ceux qui ont vécu des expériences où ils ont frôlé la mort le savent. On peut mourir comme ça, n'importe quand et

CHEZ LES ENFANTS, LA CONCEPTION DE LA MORT EST DAVANTAGE LIÉE À LA PENSÉE MAGIQUE TANDIS QUE, POUR L'ADULTE, ELLE EST EN GRANDE PARTIE LIÉE AUX APPRENTISSAGES RÉALISÉS LORS DE LA CONFRONTATION AVEC LA RÉALITÉ.

représentations. Premièrement, la mort est une violence. Michel Hanus souligne que " pour l'enfant, on ne meurt pas, on est tué $10 »$. L'enfant, en effet, éprouve des difficultés à se représenter la mort comme l'issue naturelle de toute existence. Au contraire, il voit dans la mort un accident, c'est-à-dire un événement étrange qui n'aurait jamais dû se produire. Lorsqu'une personne décède, c'est qu'une force animée d'une mauvaise intention a transgressé le cours normal des choses pour la tuer. Deuxièmement, la mort est réversible. Ce n'est que progressivement et à la suite d'un long travail sur soi que l'enfant comprend le caractère irréversible de la mort. Pour lui, le fait d'être mort n'exclut pas un retour. Combien d'adultes ont affirmé contre toute attente. Cette vérité aide les enfants à mettre en place une certaine sagesse de vie. Nombre d'enfants s'interrogent sur la vie après la mort. Un enfant qui pose ce type de question veut se réconcilier avec sa mort ou la mort d'un proche. Il cherche à l'apprivoiser, à vivre avec le fait que les gens meurent et que lui-même va mourir un jour. Dolto suggère de dire ce qu'on en sait. Pour nombre de personnes, cette réponse revient à dire qu'ils n'en savent rien. Celles-ci expliqueront que chacun a ses croyances. Peut-être que l'enfant a entendu dire que la personne morte s'en va vivre au ciel. D'autres personnes vont expliquer leurs propres croyances. D'autres vont retourner la question : "Et toi, qu'est-ce que tu en penses?» 


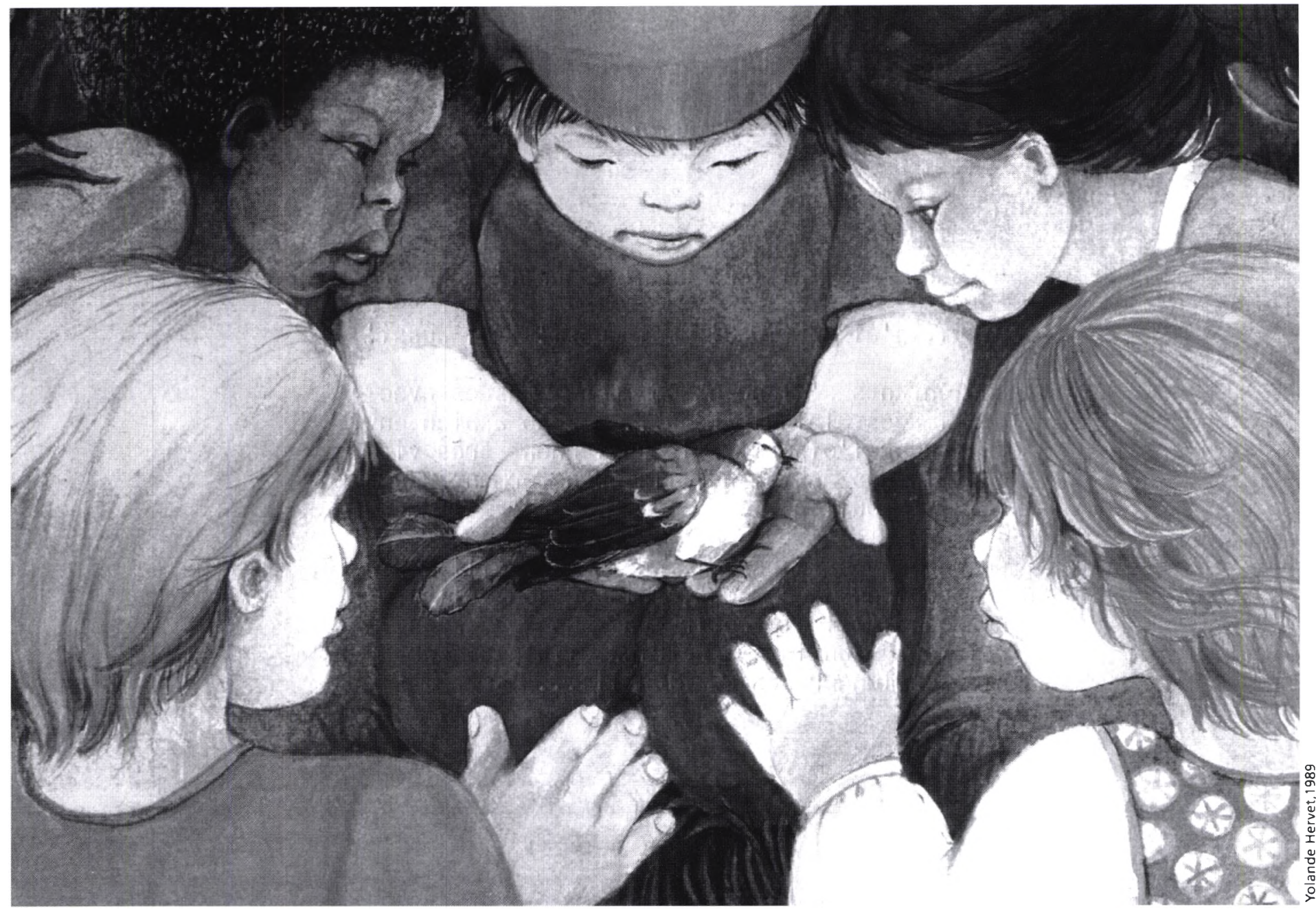

Cela permet à l'enfant de se construire sa propre conception de l'après-vie. Il est intéressant de remarquer que peu de gens répondent : «Après la mort, il n'y a rien. La personne n'existe plus ». Peutêtre qu'on ne propose pas cette réponse parce qu'au fond, elle reste inacceptable.

\section{LE RITUEL DE PARTAGE}

Ayant déjà établi un contact privilégié entre lui et ses élèves, l'enseignant est à même de leur offrir un temps d'écoute et de reconnaître leur souffrance. Il peut confirmer l'élève dans la difficulté de vivre son épreuve, lui laisser entendre qu'il est possible qu'il ait mal, que ses copains de classe souffrent comme lui. Il peut également lui signifier sa disponibilité et son écoute. Françoise Dolto ${ }^{12}$ rappelle à cet égard que toutes les paroles exprimées lors d'un décès apaisent et réconfortent. Il est bien à propos qu'un enseignant sache comment intervenir lorsque sa classe est perturbée par une perte importante. En effet, lorsqu'une telle situation se présente, le regard des élèves tend à converger vers celui de l'enseignant. Michel Hanus observe à cet égard que "le deuil des enfants se calque sur celui des adultes $» 13$.
Les personnes semblent aujourd'hui plus fragiles que jamais devant leur propre mort et celle des autres ${ }^{14}$. Nombre d'individus confrontés à la perte d'un être cher se sentent plus désemparés qu'auparavant et éprouvent plus de difficulté à trouver de l'assistance pour les soutenir. L'aide autrefois offerte par l'Église, la famille et les proches se fait maintenant plus discrète. Pour certains endeuillés, elle serait même absente. Cette fragilité accrue de l'individu devant la mort pourrait s'expliquer par la perte des rituels, par leur appauvrissement, voire même par leur disparition ${ }^{15}$. Pourquoi, de façon générale, est-il si important d'associer des rituels aux expériences de deuil ? La mise en place d'un rituel permet notamment d'aider l'endeuillé à exprimer sa souffrance d'une manière acceptable pour lui et pour les autres, de favoriser l'émergence d'un sens, de mettre des mots sur la mort et de souligner l'importance et la beauté de la vie.

Un rituel de partage est une activité lors de laquelle chaque élève est convié à partager ce qu'il ressent. Lorsque des élèves d'une classe sont confrontés à une perte importante, ce rituel peut prendre la forme d'une simple discussion, d'une prière ou d'une lecture commune que chaque élève aura l'occasion de commenter. Le partage des émotions se fera autour de différentes mises en scène qui peuvent être, entre autres, de coller au mur les dessins de l'enfant décédé, ou de construire une mosaïque avec les poèmes des enfants. Notons aussi que le deuil se fait plus facilement avec l'aide d'un objet héritage. L'objet héritage ${ }^{16}$ est un objet personnel et familier ayant appartenu à la personne décédée. Une personne confrontée à la mort d'un de ses proches a grand besoin de ce genre d'objet. Cela vaut particulièrement pour l'enfant. L'objet légué à l'enfant « est un signe de présence/absence » du défunt. C'est un héritage qui aide à apprivoiser la réalité de la perte.

Voici deux exemples de rituel de partage inspirés du livre Grandir aimer, perdre et grandir de Jean Monbourquette.

Le rituel se nomme Le cercle de la solidarité. D'abord, l'enseignant informe les élèves du drame qui vient de se produire. Il explique qu'un membre du groupe est décédé et donne quelques détails sur les circonstances du décès. 
Ensuite, avec les élèves, l'enseignant prend le temps d'aménager la classe de façon à ce que chacun puisse saisir l'importance de ce moment. Afin de rompre avec l'atmosphère habituelle de la classe, il place les pupitres et les chaises dans un coin ou change de local afin de disposer d'un espace suffisant pour organiser l'activité. Puis l'enseignant invite tous les élèves à former un cercle et à s'asseoir par terre. L'enseignant propose de prendre deux minutes de silence par respect pour le disparu. Il allume une bougie à côté d'un petit coffre ouvert au centre du cercle. Ce petit coffre est orné de la photo de la personne disparue. Cette photo constitue un objet héritage légué au groupe. Ensuite, l'enseignant invite les élèves à aller se recueillir à un endroit de leur choix dans la classe et à faire un dessin pour la personne qui vient de partir. Si les élèves sont plus âgés, l'enseignant peut demander de composer un petit mot ou un court poème. Quand les dessins ou les poèmes sont terminés, l'enseignant invite les élèves à reformer le cercle.

Il invite alors chaque élève à prendre la parole et à dire quelques mots sur le dessin ou sur le poème puis à le déposer dans le coffre. À cette occasion, l'enseignant peut utiliser un "bâton de la parole ». Le bâton de la parole est un objet que les jeunes s'échangent entre eux. Il signifie que la personne qui a le bâton demande à parler et qu'elle désire être entendue. Ceux et celles qui le désirent peuvent simplement déposer ce qu'ils ont fait dans le coffre, puis laisser la parole à leur voisin. Il importe, à cette étape du rituel, de laisser les élèves s'exprimer sans toutefois forcer personne.

Le coffre et la photo du disparu pourront être exposés pour une période de temps limitée ou pour le reste de l'année scolaire, selon le souhait des élèves de la classe. Au cours de l'année, les élèves qui le désirent peuvent faire un nouveau dessin ou composer un nouveau poème qu'ils vont déposer dans le petit coffre. À la fin de l'année, ceux qui le désirent peuvent reprendre leur mot et leur dessin. Ce mot pourra être lu lors d'une cérémonie spéciale organisée en fin d'année. Si le décès est trop récent et que le deuil n'est pas terminé, l'enseignant peut alors évoquer, pour une dernière fois, lors de son dernier cours, le souvenir du défunt.

Ce rituel fait appel à de nombreuses représentations symboliques. Le cercle formé par les élèves symbolise la solidarité et l'entraide, la photo symbolise la présence du défunt, le coffre symbolise le lieu où son corps repose. Lorsque les élèves placent les dessins et les textes qu'ils ont composés dans le coffre, c'est un peu comme s'ils les offraient au défunt. Enfin, la chandelle symbolise l'espoir d'un bonheur possible malgré le drame qui vient de se produire. Elle rappelle que, malgré la mort d'une personne aimée, la vie vaut la peine d'être vécue.

Le rituel de partage nommé L'album $d u$ souvenir constitue une variante $\mathrm{du}$ Cercle de la solidarité. Comme pour le rituel précédent, l'enseignant aménage la classe pour créer une atmosphère propice au déroulement du rituel. Il convie les élèves à former un cercle et à s'asseoir par terre. Ensuite, il allume une bougie et explique le drame qui vient de se produire en répondant aux questions des élèves. Il invite également les élèves à garder un moment de silence pour penser au jeune qui vient de mourir et à faire un dessin ou à écrire un court texte. Puis il demande aux élèves de reformer le cercle et il ajoute, auprès de la bougie, une photo du jeune défunt. Chaque élève est convié à expliquer son dessin ou à lire le court texte qu'il a composé et à le placer dans un album. Cet album appartient au groupe. Il est placé dans un endroit désigné de la classe et pourra être consulté en cours d'année. À la fin de l'année, l'enseignant organisera une dernière cérémonie en l'honneur du défunt à laquelle seront conviés tous les élèves qui désirent y participer.

Il n'existe aucun modèle précis de rituels de partage. Avec ces deux exemples, nous voulions tout simplement montrer que le rituel ne requiert pas une organisation complexe. Il serait souhaitable que chaque enseignant puisse prendre quelques minutes, si une perte importante survient dans sa classe, pour organiser une activité de ce genre permettant aux élèves d'exprimer ce qu'ils vivent, de se réconforter les uns les autres et de dire au revoir au défunt.

\section{CONCLUSION}

Dans le cas d'enfants endeuillés, personne n'est en mesure de savoir à l'avance si, par exemple, un enfant acceptera la mort de son grand-père, alors que le divorce de ses parents apparaît inconcevable. Toutefois, nous savons que chaque étape est une expérience de vie singulière, mais nécessaire. En fait, la nature du deuil et l'intensité de la souffrance de l'enfant dépendent de trois facteurs : la nature de la relation qui préexistait avant de vivre une perte, la nature de cette perte et les circonstances qui ont provoqué cette perte.
Nous avons vu que pour entamer un travail de deuil, l'enfant doit se doter d'une maîtrise suffisante de la réalité de la mort. Il doit également acquérir une représentation interne et stable de la personne en qui il a investi son affection ainsi qu'une sécurité quant à la satisfaction de ses besoins en l'absence de la personne disparue. Certes, un enfant trop jeune pour réunir ces conditions ne manque pas d'être touché par une perte et de souffrir. Pour aider l'enfant à réaliser son travail de deuil, il faudra alors plus spécifiquement prendre en compte la culpabilité, la confiance, la réaction à la perte, le bouleversement dans son mode de vie, l'expression de sa souffrance, la relation avec le défunt, le besoin d'un objet héritage, ainsi que la spécificité de certains rituels.

\section{Notes}

1 Monique SÉGUIN et Lucie FRÉCHET$\mathrm{TE}$, Le deuil, une souffrance à comprendre pour mieux intervenir, Montréal, Éditions Logiques, 1995.

2 Michel HANUS, Les deuils dans la vie, Paris, Maloine, 1994.

3 Sigmund FREUD, " Deuil et mélancolie » dans Métapsychologie, Paris, Gallimard, 1968.

4 Marie-Frédérique BACQUÉ, Le deuil à vivre, Paris, Odile Jacob, 1995.

5 Françoise DOLTO, Tout est langage, Paris, Vertiges du nord/Carrere, 1987.

6 Ibid., p. 83.

7 Michel HANUS, ibid. note 2, p. 275.

8 Sigmund FREUD, "Au-delà du principe de plaisir ", dans Essais de psychanalyse, Paris, Payot, 1987, p. 83.

9 Monique SÉGUIN et Lucie FRÉCHETTE, ibid. note 1 , p. 73 , offrent une des plus récentes rétrospectives des études sur l'évolution des représentations de la mort chez l'enfant.

10 Michel HANUS, ibid. note 2, p. 257.

11 Françoise DOLTO, Parler de la mort, Paris, Gallimard, 1988.

12 Françoise DOLTO, ibid. note 5, p. 133.

13 Michel HANUS, ibid. note 2, p. 162.

14 Patrick BAUDRY, "Le sens de la ritualité funéraire ", dans Mourir aujourd'hui : les nouveaux rites funéraires sous la direction de Marie-Frédérique BACQUÉ, Paris, Éditions O. Jacob, 1997.

15 Denis JEFFREY, Jouissance du sacré : ritualité et postmodernité, Paris, Armand Colin, 1998.

16 Jean MONBOURQUETTE, Grandir aimer, perdre et grandir, Outremont, Québec, Novalis, 1994. 